\title{
Studying the signs of pulmonary hypertension shown by CT scans and correlating data obtained with echocardiography in suspected patients of pulmonary hypertension Youssriah Y. Sabria, Iman M. Hamdya, Irene M. Sabry', Assad A. Saif Al-Hadia
}

Introduction Pulmonary arterial hypertension is a devastating disease with significant disability and mortality, and it has much higher prevalence than previously thought.

\begin{abstract}
Purpose The aim was to study the signs of pulmonary hypertension shown by computed tomography (CT) scans and correlate the data obtained with echocardiography in suspected patients with pulmonary hypertension in a trial to survey patients eligible for right heart catheterization.
\end{abstract}

\begin{abstract}
Patients and methods In this study, 50 patients underwent high-resolution CT (18 cases) or CT pulmonary angiography (32 cases) using a 4-MDCT scanner for evaluation of their pulmonary parenchyma, pulmonary vasculature, and mediastinal structures to detect different diagnostic criteria, types, causes, associations, and complications of pulmonary arterial hypertension. All the 50 patients underwent echocardiography.
\end{abstract}

Results In all cases, the main pulmonary artery (MPA) was larger than $29 \mathrm{~mm}$, and was further divided according to the size into three groups $(<35 \mathrm{~mm}=52 \%,>35<40 \mathrm{~mm}=$ $22 \%$, and $\geq 40 \mathrm{~mm}=26 \%$ ). A more than $1: 1$ relationship between the segmental pulmonary artery and the bronchus in different pulmonary lobes was seen in $78 \%$ of the cases.

\section{Introduction}

Pulmonary arterial hypertension $(\mathrm{PAH})$ is a devastating disease with significant disability and mortality, and it has a much higher prevalence than previously thought. Nowadays, the diagnosis is more clearly defined, noninvasive markers of disease severity can be widely applied, and finally, we can adopt evidence-based treatment [1].

Computed tomography (CT) offers anatomic information about the size of the pulmonary arterial tree, and echocardiography identifies the functional consequences of PAH, evaluating secondary tricuspid regurgitation. Because right-sided heart catheterization is an invasive test, there is a need for accurate noninvasive markers that can be used appropriately to stratify patients for referral for right-sided heart catheterization [2].

A CT-determined main pulmonary artery (MPA) diameter of greater than $29 \mathrm{~mm}$ demonstrates $87 \%$ sensitivity and $89 \%$ specificity for predicting $\mathrm{PAH}$. The specificity increases to $100 \%$ when an MPA diameter of greater than $29 \mathrm{~mm}$ is accompanied by findings of a segmental artery-to-bronchus ratio $(\mathrm{B} / \mathrm{A})$ greater
A more than $1: 1$ relationship between the MPA and the aorta was seen in $76 \%$ of the cases. Correlation between MPA and the mean pulmonary artery pressure measured in 50 cases showed significant correlation, with a $P$ value of up to 0.001 . According to other CT findings, the causes were divided into four groups.

Conclusion Although right heart catheterization is the gold standard for the measurement of pulmonary artery pressure, this procedure is not without risk and expense. There is a need for accurate noninvasive markers that can be used appropriately to stratify patients for referral for rightsided heart catheterization. Egypt J Broncho 2013 7:60-66 (C) 2013 Egyptian Journal of Bronchology.

Egyptian Journal of Bronchology 2013 7:60-66

Keywords: correlation, computed tomography criteria pulmonary arterial hypertension, echocardiography

aDepartments of Radiodiagnosis and ${ }^{b}$ Chest Diseases, Faculty of Medicine, Cairo University, Cairo, Egypt

Correspondence to Irene Mohamed Sabry, MD, Department of Chest Diseases, Faculty of Medicine, Cairo University, Cairo, Egypt Tel. +201224109776, Fax: 0235855428 e-mail: irene.sabry@yahoo.com

Received 22 September 2013 Accepted 27 October 2013

than $1: 1$ in three of the pulmonary lobes. When the ratio of the CT-determined pulmonary artery diameter to the aortic diameter $(\mathrm{P} / \mathrm{A})$ is greater than 1 , a strong correlation with elevated mean pulmonary artery pressure has been shown, particularly in patients less than 50 years of age. A comparison of the size of the pulmonary artery to the ascending aorta $(\mathrm{AO})$ is a practicable and easy assessment to make on CT scans $[2,3]$.

\section{Aim of the work}

The aim of this study was to study the signs of pulmonary hypertension shown by CT scans, and correlate the data obtained with echocardiography in suspected patients with pulmonary hypertension in a trial to survey patients eligible for right heart catheterization.

\section{Patients and methods}

This study included 50 patients (42 women and eight men) with suspected $\mathrm{PAH}$, their age ranging from 20 to 85 years (mean 44.62 years). Patients were referred to the radiology department of Cairo university hospital 
from the chest, ICU, internal medicine, rheumatology and cardiology departments to undergo high-resolution CT (HRCT) (18 cases) or CT pulmonary angiography (CTPA) (32 cases) for different diagnostic purposes from October 2010 until February 2012. All patients were subjected to thorough history taking and clinical examination.

\section{Laboratory studies}

Routine laboratory studies were performed on patients (e.g. complete blood count). For those who underwent CTPA, a kidney function test was performed (urea and serum creatinine).

\section{Echocardiography}

Transthoracic echocardiography was performed using an HP 7500 or 5500 model (Hewllet - Packard Andover, Massachussettes, USA). Mean pulmonary artery pressure (mPAP) was measured using standard techniques. All the 50 patients underwent echocardiography.

\section{Other investigations}

Other investigations were conducted according to the patient's condition: for example pulmonary function test, lung biopsy, collagen profile, arterial blood gases, HIV test, tests to exclude causes of pulmonary hypertension other than idiopathic type of pulmonary hypertension.

Multidetector CT (MDCT) examination: all patients underwent either HRCT or CTPA examination. The procedure was conducted using a multidetector row helical scanner with four detector arrays (Light Speed Plus; GE medical systems, Milwaukee, Wisconsin, USA) in the radiology department, Cairo University.

\section{Data analysis and interpretation}

In each case, the following were considered.

In this study, guided by Frazier et al. [4], Grosse and Grosse [5], and Peña et al. [6], we used the following criteria to diagnose and assess $\mathrm{PAH}$.

The widest diameter of MPA at the site of bifurcation was measured, which was divided into:
(1) $\leq 3.5 \mathrm{~cm}$.
(2) $>3.5-<4 \mathrm{~cm}$.
(3) $\geq 4 \mathrm{~cm}$.

The relationship between segmental pulmonary arteries and the bronchus in different lung lobes was determined, which was divided into:

(1) Normal (when it is = 1:1 in all lobes).

(2) One (when it is $>1: 1$ in one lobe).
(3) Two (when it is $>1: 1$ in two lobes).

(4) Three (when it is $>1: 1$ in three lobes).

The relationship between MPA and the adjacent AO was determined, which was divided into:

(1) Normal (when it is $<1: 1$ ).

(2) Increased (when it is $>1: 1$ ).

Other CT signs that were considered as a cause, association or complication of $\mathrm{PAH}$ were divided into:

(1) Lung parenchyma

(2) Pleural

(3) Mediastinal

(4) Vascular

(5) Cardiac and pericardial findings

\section{Results}

In this study, 50 patients underwent HRCT (18 cases) or CTPA (32 cases), using a 4-MDCT scanner for evaluation of their pulmonary parenchyma, pulmonary vasculature and mediastinal structures to detect different diagnostic criteria, types, causes, associations and complications of $\mathrm{PAH}$.

The study included 42 women and eight men, their age ranging from 20 to 85 years (mean 44.62 years).

\section{Groups of the study}

The study population was divided according to the suspected causes of pulmonary hypertension into four groups:

Group A including patients with PAH due to pulmonary causes (corpolmonale),

Group B including patients with PAH due to cardiac causes,

Group $\mathrm{C}$ including patients with $\mathrm{PAH}$ due to pulmonary embolism (PE) and

Group D including patients with PAH due to unknown causes (Tables 1-16).

\section{Discussion}

$\mathrm{PAH}$ is a challenge for clinicians and radiologists, with a variety of possible underlying causes, each with its own specific treatment. MDCT can play a vital role in elucidating underlying cardiac, vascular, and pulmonary causes [7]. 
Table 1 Measurement of MPA diameter in all cases (50 cases)

\begin{tabular}{lccc}
\hline MPA diameter & Men & Women & Total \\
\hline$\leq 3.5 \mathrm{~cm}$ & 4 & 22 & 26 \\
$>3.5<4 \mathrm{~cm}$ & 1 & 0 & 11 \\
$\geq 4 \mathrm{~cm}$ & 3 & 10 & 13 \\
\hline
\end{tabular}

MPA, main pulmonary artery.

Table 2 Relationship between segmental pulmonary arteries and bronchi in all cases (50 cases)

\begin{tabular}{lccr}
\hline $\begin{array}{l}\text { Relationship between segmental } \\
\text { pulmonary arteries and bronchus }\end{array}$ & Men & Women & Total \\
\hline Normal $(=1: 1)$ & 3 & 8 & 11 \\
$>1: 1$ in one lobe & 0 & 6 & 6 \\
$>1: 1$ in two lobe & 2 & 4 & 6 \\
$>1: 1$ in three lobe & 3 & 24 & 27 \\
\hline
\end{tabular}

Table 3 Relationship between MPA and adjacent AO in all cases (50 cases)

\begin{tabular}{lccc}
\hline $\begin{array}{l}\text { Relationship between MPA and } \\
\text { aorta }\end{array}$ & Men & Women & Total \\
\hline Normal $(<1: 1)$ & 3 & 13 & 17 \\
Increased $(>1: 1)$ & 5 & 29 & 33 \\
\hline
\end{tabular}

AO, aorta; MPA, main pulmonary artery.

Table 4 Echocardiography results (positive = $\mathrm{PAH}$, negative = no $\mathrm{PAH})$ in all cases (50 cases)

\begin{tabular}{lccc}
\hline Echocardiography result & Men & Women & Total \\
\hline+ & 6 & 34 & 40 \\
- & 1 & 9 & 10 \\
\hline
\end{tabular}

$\mathrm{PAH}$, pulmonary arterial hypertension.

Table 5 Mean value of MPA measured by CT (50 cases)

\begin{tabular}{cccccc}
\hline & $N$ & Minimum & Maximum & Mean & SD \\
MPA & 50 & 1.46 & 5.72 & 3.6338 & 0.67422 \\
\hline
\end{tabular}

CT, computed tomography; MPA, main pulmonary artery.

Table 6 Mean value of MPA in positive PAH cases by echo (40 cases)

\begin{tabular}{cccccc}
\hline & $N$ & Minimum & Maximum & Mean & SD \\
MPA & 40 & 3.02 & 5.72 & 3.7683 & 0.62899 \\
\hline
\end{tabular}

MPA, main pulmonary artery; $\mathrm{PAH}$, pulmonary arterial hypertension.

Table 7 Correlation between echocardiography results and MPA measurement (positive = PAH, negative = no PAH)

\begin{tabular}{lccc}
\hline & \multicolumn{2}{c}{ Echocardiography results } & \\
\cline { 2 - 3 } MPA measurement & + & - & Total \\
\hline$\leq 3.5 \mathrm{~cm}$ & 16 & 0 & 26 \\
$>3.5<4 \mathrm{~cm}$ & 11 & 0 & 11 \\
$\geq 4 \mathrm{~cm}$ & 13 & 0 & 13 \\
\hline
\end{tabular}

MPA, main pulmonary artery; $\mathrm{PAH}$, pulmonary arterial hypertension.

The clinical classification system for PAH was updated at the fourth World Symposium on PAH in Dana Point, California, in 2008. In patients with suspected $\mathrm{PAH}$, the diagnostic approach includes four stages:
Table 8 Mean value of mPAP measured by echo in positive PAH cases (40 cases)

\begin{tabular}{lccccc}
\hline & $N$ & Minimum & Maximum & Mean & SD \\
Echo (PAP) & 40 & 30 & 118 & 60.65 & 25.017
\end{tabular}

mPAP, mean pulmonary artery pressure; $\mathrm{PAH}$, pulmonary arterial hypertension; PAP, pulmonary artery pressure.

Table 9 Correlation between MPA and mPAP measured in 50 cases

\begin{tabular}{lc}
\hline & mPAP \\
\hline MPA & \\
Correlation coefficient & 0.448 \\
$P$ value & 0.001 \\
$N$ & 50 \\
\hline
\end{tabular}

Strong statistically significant correlation between MPA and mPAP in all cases with a $P$ value of 0.001 .; MPA, main pulmonary artery; mPAP, mean pulmonary artery pressure.

Table 10 Correlation between MPA and mPAP measured in 40 cases

\begin{tabular}{lc}
\hline & mPAP \\
\hline MPA & \\
Correlation coefficient & 0.312 \\
P value & 0.048 \\
$N$ & 40 \\
\hline
\end{tabular}

Statistically significant correlation between MPA and mPAP measured by echo in positive PAH cases.; MPA, main pulmonary artery; mPAP, mean pulmonary artery pressure.

Table 11 Evaluation of different parameters: sensitivity, specificity and positive predictive value for (MPA>29 $\mathrm{mm}$ ), (MPA > $29 \mathrm{~mm}+\mathrm{P} / \mathrm{A}>1$ ) and (MPA > $29 \mathrm{~mm}+\mathrm{P} / \mathrm{A}>1+$ $B / A>1$ in three lobes) in CT scans

Variables TPFN FP TN Sensitivity Specificity Positive (\%) (\%) predictive value (PPV)

(\%)

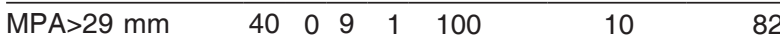

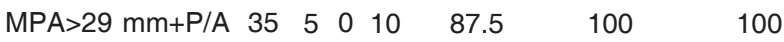

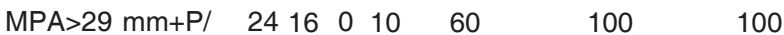
$A+B / A$ in three

lobes

Sensitivity $=\frac{T P}{T P+F N} \quad$ Specificity $=\frac{T N}{T N+F P}$ Positive predictive value $(\mathrm{PPV})=\frac{\mathrm{TP}}{\mathrm{TP}+\mathrm{FN}} ; \mathrm{B} / \mathrm{A}$, artery-to-bronchus ratio; $\mathrm{CT}$,

computed tomography; FN, false negative; FP, false positive; MPA, main pulmonary artery; $\mathrm{P} / \mathrm{A}$, pulmonary artery diameter to the aortic diameter; TN, true negative; TP, true positive.

suspicion, detection, classification, and functional evaluation [6].

In the current study, 50 patients ( 42 women and eight men) were examined by either HRCT or CTPA using a 4-MDCT scanner in the radiology department, Cairo university hospital.

The study population was divided according to clinical, echo, and MDCT findings into four groups: group A including patients with $\mathrm{PAH}$ due to pulmonary causes, 
Table 12 Correlation between echocardiography results and the relationship between the segmental pulmonary artery and the bronchus (positive $=$ PAH, negative $=$ no PAH)

\begin{tabular}{lrrr}
\hline & \multicolumn{2}{c}{ Echocardiography result } & \\
\cline { 2 - 2 } $\begin{array}{l}\text { Relationship between } \\
\text { segmental pulmonary }\end{array}$ & + & - & Total \\
artery and bronchus & & & \\
\hline Normal $(1: 1)$ & 3 & 8 & 11 \\
$>1: 1$ in one lobe & 5 & 1 & 6 \\
$>1: 1$ in two lobe & 5 & 1 & 6 \\
$>1: 1$ in three lobe & 27 & 0 & 27 \\
$(r=0.126, P>0.005)$ & & &
\end{tabular}

$\mathrm{PAH}$, pulmonary arterial hypertension.

Table 13 Correlation between echocardiography results and the relationship between the pulmonary artery and $A O$ (positive $=\mathbf{P A H}$, negative $=$ no $\mathrm{PAH})$

\begin{tabular}{|c|c|c|c|}
\hline \multirow{2}{*}{$\begin{array}{l}\text { Relationship between } \\
\text { MPA and aorta }\end{array}$} & \multicolumn{2}{|c|}{ Echocardiography result } & \multirow[t]{2}{*}{ Total } \\
\hline & + & - & \\
\hline Normal $(<1: 1)$ & 7 & 10 & 17 \\
\hline Increased (>1: 1) & 33 & 0 & 33 \\
\hline
\end{tabular}

Odds ratio $=2.429,95 \% \mathrm{Cl}(1.376-4.286)$. A statistically significant association was found between the presence of pulmonary hypertension measured by echo and elevated MPA/A ratio $>1$. $\mathrm{AO}$, aorta; $\mathrm{Cl}$, confidence interval; MPA, main pulmonary artery; $\mathrm{PAH}$, pulmonary arterial hypertension.

Table 14 Different CT findings in all cases

\begin{tabular}{lc}
\hline Findings & Frequency \\
\hline Parenchymal presentation \\
Coarse reticulation & 11 \\
Consolidation & 10 \\
Mosaic attenuation/normal lungs & 6 \\
Ground glass & 5 \\
Emphysema & 3 \\
Peribronchial thickening & 2 \\
Atelectatic bands & 3 \\
Fibrosis/centrilobular nodules/ & 3 \\
bronchiectasis/cavitary lesion & \\
Pulmonary nodules/hyperinflated & 2 \\
lung/lobar collapse & \\
Tree in bud/bullae/diffuse cystic & 1 \\
lesion/hilar mass & \\
Pleural & \\
Pleural effusion & 8 \\
Pleural thickening & 1 \\
Cardiac & \\
Multichambers cardiomegaly & \\
Left atrial enlargement & \\
Vascular & \\
Pulmonary artery filling defect & \\
Mediastinal & \\
Lymph nodes & \\
Thymus gland enlargement/patulous & \\
esophagus & 5 \\
\hline
\end{tabular}

$\mathrm{CT}$, computed tomography.

group B including patients with $\mathrm{PAH}$ due to cardiac causes, group $\mathrm{C}$ including patients with $\mathrm{PAH}$ due to $\mathrm{PE}$, and group D including patients with $\mathrm{PAH}$ due to unknown causes (idiopathic) (Table 16).
Table 15 Associations with PAH

\begin{tabular}{lc}
\hline Complications & Number of presentation \\
\hline Right-sided cardiomegaly & 23 \\
Pericardial effusion & 12 \\
Pericardial thickening & 9 \\
\hline
\end{tabular}

$\mathrm{PAH}$, pulmonary arterial hypertension.

Table 16 Total number of patients in each group

\begin{tabular}{lc}
\hline Groups & $\%$ \\
\hline Group A & 36 \\
Group B & 32 \\
Group C & 22 \\
Group D & 10 \\
Total & 100 \\
\hline
\end{tabular}

This classification is highly matching with the classification adopted in the Fourth World Symposium on PAH: perspectives for practice 2008 [8].

In this study, guided by Frazier et al. [4], Grosse and Grosse [5], and Peña et al. [6], we used the following criteria to diagnose and assess PAH.

\section{Measuring of MPA}

The measurement of MPA was the first criterion we used in the CT diagnosis of PAH. This was also the first criterion used in the diagnosis of pulmonary hypertension in the study conducted by Devaraj et al. [9], Grosse and Grosse [5], and Peña et al. [6].

Grosse and Grosse [5] stated that a pulmonary artery with a diameter more than $29 \mathrm{~mm}$ has a positive predictive value of $97 \%$, a sensitivity of $87 \%$ and a specificity of $89 \%$ for diagnosing $\mathrm{PAH}$.

However, in all our cases, the MPA was more than $29 \mathrm{~mm}$ in its widest dimension. For easier assessment and correlation, this was further divided into three categories. This division was derived from the study conducted by Devaraj et al. [9]. In our study, the MPA diameter was measured at the workstation using an electronic caliper.

The first category is $\leq 3.5 \mathrm{~cm}(n=26,52 \%)$.

The second category is between $>3.5$ and $<4 \mathrm{~cm}(n=$ $11,22 \%)$.

The third category is $\geq 4 \mathrm{~cm}(n=13,26 \%)$.

The first category is the largest; this result was likely because our cases mostly presented early, whereas the third category is the smallest as most of the cases in the third category are in advanced stage of the disease (Table 1). 


\section{The relationship between segmental pulmonary arteries and bronchi}

The relationship between the segmental pulmonary arteries and their neighboring bronchi is the second criterion that we checked. In this criterion, we compared the measurement of the segmental pulmonary artery with its neighboring bronchus, which is normally $1: 1$. When the pulmonary blood pressure increases, this ratio also increases and the number of lobes in which these relations are present also increases.

The enlargement of the segmental arteries, measured by making a subjective comparison with the size of the adjacent bronchus, occurs in patients with PAH [10]. The study conducted by Devaraj et al. [9] suggests that the segmental arterial size is a reliable marker of mPAP, and in practice, the comparison with the adjacent bronchus is more convenient.

According to Grosse and Grosse [5] and Peña et al. [6], pulmonary hypertension can be predicted reliably when the $\mathrm{CT}$ demonstrated a diameter of the MPA greater than $29 \mathrm{~mm}$ and the segmental B/A is greater than $1: 1$ in three of four pulmonary lobes (specificity 100\%).

The presence of this relationship in three lobes was seen in $54 \%(n=27)$ of our cases; however, this relationship was seen only in two lobes in $12 \%(n=6)$ of the cases, and in one lobe in $12 \%(n=6)$ of the cases. Normal $\mathrm{B} / \mathrm{A}$, however, was found in only $22 \%(n=11)$ of the cases, all of which were of the first category (MPA diameter $\leq 3.5 \mathrm{~cm})($ Table 2).

\section{The relationship between MPA and AO}

The third criterion is the relationship between the MPA and the AO. The AO is the largest artery in the body, and normally, the MPA is smaller in diameter than the AO. When there is an increase in the pulmonary pressure, the MPA artery diameter may become larger than that of the $\mathrm{AO}$ and the relationship becomes more than $1: 1$.

Devaraj et al. [9] suggested that the ratio of the diameter of the MPA to the diameter of the AO is an accurate marker of mPAP because confounding variables, such as patient size, influence the size of the pulmonary artery and the ascending $\mathrm{AO}$ equally, enabling a form of 'internal correction'.

In this study, an increase in the ratio between MPA and $\mathrm{AO}$ is seen in $76 \%(n=33)$ of cases.

The ratio was normal in $24 \%(n=17)$ of our cases. All of them were older than 45 years, and the measurement of MPA was less than $3.5 \mathrm{~cm}$ (Table 3).
The results in this study were correlated with the echocardiography results, in which $80 \%(n=40)$ of the patients who underwent echocardiography had $\mathrm{PAH}$ (positive) and 20\% $(n=10)$ were normal (negative) (Table 4). In all negative cases, the MPA diameter was less than $3.5 \mathrm{~cm}$.

A normal segmental pulmonary B/A relationship was seen in $7.5 \%(n=3)$ of the positive cases and $67 \%$ $(n=8)$ of the negative cases. In the rest of the normal cases [the remaining 33\% $(n=3)$ ], the segmental pulmonary artery was enlarged compared with the adjacent bronchus in one lobe (one case) and two lobes (one case), but no cases showed three lobes being affected (Table 12).

A normal relationship between MPA and AO was seen in $14 \%(n=7)$ of the positive echocardiography cases.

The mean value of MPA as measured by CT in 50 cases was $3.6338 \pm 0.67422$ (Table 5).

The mean value of MPA as measured by echocardiography in 40 positive $\mathrm{PAH}$ cases was $3.7683 \pm 0.62899$ (Table 6).

The mean value of mPAP in positive PAH (40 cases) was $60.65 \pm 25.017$ (Table 8).

The correlation between MPA and mPAP measured in 50 cases showed significant correlation, with a $P$ value of up to 0.001 (Table 9).

The correlation between MPA and mPAP measured in 40 cases (positive pulmonary hypertension) showed a strongly statistically significant correlation between MPA and PAP as measured by echocardiography (Table 10).

From the correlation of our CT findings in this study with the echocardiography results (Table 11), pulmonary hypertension can be predicted in $82 \%$ of the cases depending on the measurement of MPA alone, in $100 \%$ of the cases if there is also an associated increase in the relationship between MPA and AO (regardless of the age) and in 100\% of the cases if there is an associated increase in the ratio between segmental pulmonary arteries and the bronchus in at least one lobe (Table 11).

The study conducted by Devaraj et al. [9] showed that a combination of $\mathrm{CT}$ and echocardiographic markers of $\mathrm{PAH}$ in the form of a composite index is more strongly related to $\mathrm{mPAP}$ than either test in isolation.

The main complication of PAH is enlargement of the right ventricle in comparison with the left ventricle, 
which is seen in $47 \%(n=23)$ of the cases as signs of failure or impending failure.

The most clinically relevant long-term complication that affects survival in patients with $\mathrm{PAH}$ is right ventricular dysfunction leading to right heart failure [11].

Right heart enlargement can be assessed qualitatively by comparing its size with the corresponding left heart. At the mid-plane of the left ventricle, the internal diameter of the right ventricle is usually equal to or slightly less than that of the left ventricle [12]. The radiological sign of heart failure in $\mathrm{CT}$ as the dilatation of the inferior vena cava (IVC) was seen in two cases that underwent CTPA (as contrast delineates the IVC).

Dilatation of the IVC and hepatic veins and pericardial effusion are also mentioned as signs of right-sided heart failure [13].

Pericardial effusion was present alone in 12 cases and pericardial thickening in nine cases; both were present together in two cases.

In this study, 18 patients were included in group A (Table 16). They were clinically diagnosed as pulmonary hypertension and were all referred from different hospital departments to undergo either HRCT or CTPA to detect the underlying lung etiology.

This group represents $36 \%(n=18)$ of the study. In this group, we found diffuse bilateral lung lesions. The main HRCT findings were bilateral coarse reticulation, bilateral ground glass opacity, bilateral fibrosis and emphysematous changes. According to Grosse and Grosse [5], lung disease is the most common cause of PAH.

On correlating our results with the study conducted by Devaraj et al. [9] interstitial lung disease causing PAH represents $50 \%$ of their 77 cases; however, cardiac causes were not included in their study. In their study, idiopathic pulmonary fibrosis (IPF) represented 70\% of the interstitial lung disease causing PAH. In our study, IPF had an upper hand in the diagnosis $(83.3 \%$; $n=15)$ of the cases in group A.

Group B in this study includes 16 patients (Table 16). They were all referred from different hospital departments to undergo either HRCT or CTPA. They were all proved to have cardiac causes by echocardiography (diastolic and systolic heart disease, valvular heart disease, and congenital heart disease).

According to Zeeb and Green [12], CT can be useful for analyzing cardiac chambers to determine their size, shape, and thickness. Normal left atrium should have an anteroposterior dimension of $\sim 4.5 \mathrm{~cm}$. Our cardiac cases were due to congenital cardiac lesion $(25 \% ; n=4)$, and they were diagnosed by echocardiography. One had patent ductus arteriosus with a patent foramen ovale, the second one had ventricular septal defect and two cases had patent ductus arteriosus only. All these cases had left to right shunt. Grosse and Grosse [5] mentioned that PAH due to sustained cardiac leftto-right shunt can be seen in patients with congenital cardiac abnormalities such as ventricular septal defect, atrial septal defect, and patent ductus arteriosus.

The third group (group C) included 11 patients. They were all referred from different hospital departments to undergo mainly CTPA. They were suspected of having PAH due to PE. Eight of them had Doppler of lower limbs showing deep venous thrombosis.

This group represented 22\% $(n=11)$ of the study. According to Wildberger et al. [14], four-slice MDCT showed promising results regarding $\mathrm{PE}$ diagnosis. From a radiological point of view, CTPA has effectively became the first imaging test in clinical routine, as patients with a high-quality negative CTPA do not require further examination or treatment for suspected PE.

In all these cases, vascular and parenchymal signs of $\mathrm{PE}$ were evaluated. The main vascular sign was filling defect in the pulmonary artery. The main parenchymal CT radiological manifestation was multiple subpleural wedge-shaped consolidation representing pulmonary infarctions and mosaic perfusion.

\section{Conclusion}

Although right heart catheterization is the gold standard for the measurement of pulmonary artery pressure, this procedure is not without risk and expense. As a result, noninvasive estimation of MPAP is of particular interest.

From the correlation of our CT findings in this study with the echocardiography results, PAH can be predicted in $82 \%$ of the cases depending on the measurement of MPA alone, in $100 \%$ of the cases, if there is also an associated increase in the relationship between MPA and the AO (regardless of the age) and in $100 \%$ of the cases if there is an associated increase in the ratio between segmental pulmonary arteries and the bronchus in at least one lobe.

As a noninvasive test, $\mathrm{CT}$ is performed routinely in patients being investigated for a possible diagnosis 
of PAH. It also has the potential to provide the first diagnosis of the condition. Echocardiography is usually more relied upon to identify $\mathrm{PAH}$, although it can also substantially lead to an underestimation or overestimation of systolic pulmonary arterial pressure. Using tests in combination is more powerful in determining increased pulmonary arterial pressure. Echocardiography is a frequently used noninvasive screening tool that provides an estimate of systolic pulmonary arterial pressure. $\mathrm{CT}$ and echocardiography provide complementary information regarding $\mathrm{PAH}$.

\section{Acknowledgements Conflicts of interest}

None declared.

\section{References}

1 Wu SC, Caravita S, Lisi E, Pierini S, Dadone V, Todd SE, et al. Pulmonary arterial hypertension. Intern Emerg Med 2009; 4:459-470.

2. Ng CS, Wells AH, Padley SPA. CT sign of chronic pulmonary arterial hypertension, the ratio of main pulmonary artery to aortic diameter. $J$ Thorac Imaging 1999; 1:270-278.

3. Devaraj A, Wells AU, Meister MG, Corte TJ, Hansell DM. The effect of diffuse pulmonary fibrosis on the reliability of CT signs of pulmonary hypertension radiology. Radiology 2008; 249:1042-1049.
4 Frazier AA, Galvin JR, Franks TJ, Rosado-De-Christenson ML. Pulmonary vasculature: hypertension and infarction. Radiographics 2000; 20:491-524.

5 Grosse C, Grosse A. CT findings in diseases associated with pulmonary hypertension: a current review. Radiographics 2010; 30:1753-1777.

6 Peña E, Dennie C, Veinot J, Muñiz SH. Pulmonary hypertension: how the radiologist can help. Radiographics 2012; 32:9-32.

7 Tsai IC, Tsai WL, Wang KY, Chen MC, Liang KW, Tsai HY, et al. Comprehensive MDCT evaluation of patients with pulmonary hypertension: diagnosing underlying causes with the updated dana point 2008 classification. Am J Roentgenol 2011; 197:W471-W481.

8 Robbins IM. Epidemiology and classification of pulmonary hypertension. Advances in pulmonary hypertension. Official J Pulmonary Hypertens Assoc 2009; 8:77-78.

9 Devaraj A, Wells AU, Meister MG, et al. Detection of pulmonary hypertension with multidetector CT and echocardiography alone and in combination. Radiology 2010; 254:609-616.

10 Pérez-Enguix D, Morales P, Tomás JM, Vera F, Lloret RM. Computed tomographic screening of pulmonary arterial hypertension in candidates for lung transplantation. Transplant Proc 2007; 39:2405-2408.

11 Voelkel NF, Quaife RA, Leinwand LA, Barst RJ, McGoon MD, Meldrum $\mathrm{DR}$, et al. Right ventricular function and failure. Report of a National Heart, Lung, and Blood Institute working group on cellular and molecular mechanisms of right heart failure. Circulation 2006; 114:1883-1891.

12 Zeeb LM, Green CE. Detecting cardiac abnormalities on routine chest CT. Appl Radiol 2009; 38:28-37.

13 van der Meer RW, Pattynama PM, van Strijen MJ, van den Berg-Huijsmans AA, Hartmann IJ, Putter $\mathrm{H}$, et al. Right ventricular dysfunction and pulmonary obstruction index at helical CT: prediction of clinical outcome during 3-month follow-up in patients with acute pulmonary embolism. Radiology 2005; 235:798-803.

14 Wildberger JE, Mahnken AH, Das M, Küttner A, Lell M, Günther RW. CT imaging in acute pulmonary embolism: diagnostic strategies. Eur Radiol 2005; 15:919-929. 